\title{
POISSON SMOOTH STRUCTURES ON STRATIFIED SYMPLECTIC SPACES
}

\author{
HÔNG VÂN LÊ, PETR SOMBERG AND JIŘI VANŽURA
}

\begin{abstract}
In this paper we introduce the notion of a smooth structure on a stratified space, the notion of a Poisson smooth structure and the notion of a weakly symplectic smooth structure on a stratified symplectic space, refining the concept of a stratified symplectic Poisson algebra introduced by Sjamaar and Lerman. We show that these smooth spaces possess several important properties, e.g. the existence of smooth partitions of unity. Furthermore, under mild conditions many properties of a symplectic manifold can be extended to a symplectic stratified space provided with a smooth Poisson structure, e.g. the existence and uniqueness of a Hamiltonian flow, the isomorphism between the Brylinski-Poisson homology and the de Rham homology, the existence of a Leftschetz decomposition on a symplectic stratified space. We give many examples of stratified symplectic spaces possessing a Poisson smooth structure which is also weakly symplectic.
\end{abstract}

\section{Contents}

1. Introduction

2. Stratified spaces and their smooth structures

2.1. Stratified spaces

2.2. Smooth structure on stratified spaces and its simplest properties

2.3. Examples of smooth structures on stratified spaces

3. Symplectic stratified spaces and compatible smooth structures 10

3.1. Symplectic stratified spaces

3.2. Weakly symplectic smooth structures and Poisson smooth structures 11

3.3. The existence of Hamiltonian flows $\quad 15$

3.4. Brylinski-Poisson homology $\quad 16$

3.5. A Leftschetz decomposition 17

4. Conclusions 20

References $\quad 20$

AMSC: 51H25, 53D05, 53D17

Key words: $C^{\infty}$-ring, stratified space, symplectic form, Poisson structure 


\section{INTRODUCTION}

Many classical problems on various classes of topological spaces reduce to the quest for its appropriate functional structure. Examples of topological spaces we are interested in comprise stratified spaces equipped with an additional structure of geometrical origin. Due to the lack of canonical notion of the sheaf of smooth (or analytic) functions on such spaces one has to define a smooth structure with all derived smooth (or analytical) notions such that the obtained smooth structure satisfies good formal properties.

In this paper we study smooth structures on stratified spaces, developing the ideas in [16]. We observe that many properties of smooth structures on pseudomanifolds with isolated conical singularities also hold for a larger class of locally trivial spaces with singularities with cone as typical fiber, if one poses a mild, natural, local condition on these smooth structures (Definition 2.5). In this extension we have to take care of (possibly disconnected) regular strata of different dimensions. A large part of our note concerns with compatible smooth structures on stratified symplectic spaces. Stratified symplectic spaces appear abundantly in geometry and mathematical physics [13, [14, [15]. They are subjects of intensive study in symplectic geometry since nineties [25], 2], [5], [27, [13]. In [1, 7] and many other papers in this direction the authors considered complex manifolds $M_{\mathbb{C}}^{2 n}$ with a holomorphic symplectic form $\omega^{2}$, which can be turned into a symplectic form $\tilde{\omega}^{2}$ on differentiable manifolds $M_{\mathbb{C}}^{2 n}$ of real dimension $4 n$ by setting $\tilde{\omega}^{2}:=\operatorname{Re}\left(\omega^{2}\right)+\operatorname{Im}\left(\omega^{2}\right)$. The theory of smooth structures on general stratified symplectic spaces has been introduced by Sjamaar and Lerman [25], then developed by many others [2], 27], [13] (in Remarks 2.7],3, 3.2 and Example [3.5] we compare our notion of a smooth structure on a stratified symplectic space with that given by Sjamaar-Lerman). Pflaum introduced smooth structures on stratified spaces by means of a maximal atlas (Remark 2.7.3), and with reference to a smooth structure, he developed extensions to stratified spaces of standard differential geometric concepts [27. Huebschmann refined the notion of symplectic stratified spaces by introducing the notion of stratified polarization [13. Our axiomatic approach to the notion of a smooth structure uses heavily the notion of $C^{\infty}$-algebras defined in [21]. We refer the reader to [11] for the notion of $C^{\infty}$ differentiable space developed by Gonsalez and de Salas, which also grew from the notion of $C^{\infty}$-algebra, but they did not treat stratified spaces. In our study we discover the existence of a class of symplectic stratified spaces which can be supplied with a Poisson and weakly symplecitc smooth structure. This class is large enough to encompass basic examples of symplectic singular spaces considered in 25] (Example 3.5. Proposition 3.8) as well as many important singularities on the closure of adjoint orbits of nilpotent elements of complex Lie algebras (Example 3.9. Proposition 3.11).

The structure of this paper is as follows. In chapter 2 we introduce the notion of a smooth structure on a stratified space (Definition 2.5) and compare our concept of a smooth structure with some other concepts (Remarks 2.72-3). We prove that a smooth stratified space possesses several important properties, e.g. the existence of smooth partitions of unity (Lemma 2.6. Proposition 2.8 and Corollary 2.9), which will be needed in later sections (Remarks 3.142, 3.22). In chapter 3 we study natural smooth structures on stratified symplectic spaces (Definition 3.3). We show that, under mild conditions, stratified symplectic spaces $(X, \omega)$ 
equipped with a Poisson smooth structure possess a variety of basic properties of smooth symplectic manifolds, e.g. the existence and uniqueness of a Hamiltonian flow, the isomorphism between the Brylinski-Poisson homology and the de Rham homology, and the existence of a Leftschetz decomposition (Theorems 3.16, 3.13 3.21. Proposition 3.20). We also show many examples of weakly symplectic smooth structures and Poisson smooth structures (Examples 3.5, 3.9).

\section{Stratified SPaCes and their Smooth structures}

In this section we recall the notion of a stratified space following Goresky's and MacPherson's concept [10, p.36], 25, §1] (Definition [2.2). Then we introduce the notion of a smooth structure on a stratified space (Definition 2.5). Our concept of a smooth structure on a stratified space is a natural extension of our concept of a smooth structure on a pseudomanifold with isolated conical singularities given in [16, §2], using the key notion of a product smooth structure (Remark [2.72). (The notion of a product smooth structure has been introduced by Mostow in [23, $\S 3]$ and has been used by many authors). We prove several important properties of a smooth structure on a stratified space, e.g. the existence of smooth partitions of unity and its consequences (Lemma 2.6. Proposition 2.8, Corollary 2.9), the existence of a locally smoothly contractible, resolvable smooth structure on pseudomanifolds with edges (Lemma 2.16). We show that a resolvable smooth structure satisfying a mild condition is not finitely generated (Proposition 2.17). We introduce the notion of a smooth differential form and a smooth Zariski vector field (Definition 2.10). We compare our concept of a smooth structure with some other concept (Remark 2.7,3).

2.1. Stratified spaces. We begin with the notion of a decomposed space.

Definition 2.1. ([10, p.36], [25, Definition 1.1]) Let $X$ be a Hausdorff and paracompact topological space and let $\mathcal{S}$ be a partially ordered set with ordering denoted by $\leq$. An $\mathcal{S}$-decomposition of $X$ is a locally finite collection of disjoint locally closed manifolds $S_{i} \subset X$ (one for each $i \in \mathcal{S}$ ) called strata such that

1) $X=\cup_{i \in \mathcal{S}} S_{i}$;

2) $S_{i} \cap \bar{S}_{j} \neq \emptyset \Longleftrightarrow S_{i} \subset \bar{S}_{j} \Longleftrightarrow i \leq j$.

We define the depth of a stratum $S$ as follows

$$
\operatorname{depth}_{X} S:=\sup \left\{n \mid \text { there exist strata } S=S_{0}<S_{1}<\cdots<S_{n}\right\} .
$$

We define the depth of $X$ to be the number depth $X:=\sup _{i \in \mathcal{S}} \operatorname{depth}_{X} S_{i}$. The dimension of $X$ is defined to be the maximal dimension of its strata.

As in 25] we consider only finite-dimensional decomposed spaces $X$. We call a stratum $S \subset X$ singular, if there is another stratum $S^{\prime} \subset X$ such that $S \subset \bar{S}^{\prime}$. Otherwise $S$ is called regular. We call

$$
X^{\text {reg }}:=\{\cup S \mid S \text { is a regular stratum of } X\}
$$

the regular part (component) of $X$. Clearly $\overline{X^{r e g}}=X$.

Now we specify a subclass of decomposed spaces consisting of locally trivial spaces with cone as typical fiber. Recall that a cone $c L$ over a topological space $L$ is the topological space $L \times[0, \infty) / L \times\{0\}$. Let $[z, t]$ denote the image of $(z, t)$ in a cone $c L$ under the projection $\pi: L \times[0, \infty) \rightarrow c L$. We denote by $c L(\varepsilon)$ the open subset $\{[z, t] \in c L \| t<\varepsilon\}$. 
Definition 2.2. ([9], 25, Definition 1.7]) A decomposed space $X$ is called a stratified space if the strata of $X$ satisfy the following condition defined recursively. Given a point $x$ in a stratum $S$ there exist an open neighborhood $U(x)$ of $x$ in $X$, an open ball $B_{x}$ around $x$ in $S$, a compact stratified space $L$, called the link of $x$, and a homeomorphism $\phi_{x}: U(x) \rightarrow B_{x} \times c L(1)$ that preserves the decomposition.

Remark 2.3. 1. It is well-known that a Whitney stratified subspace in $\mathbb{R}^{n}$ is a stratified space in sense of Definition 2.2 [19.

2. In the literature there are different concepts of a stratified space, see e.g. [27. Chapter 1] for a discussion.

Example 2.4. 1. Among important examples of stratified spaces of depth 1 are pseudomanifolds with edges, see e.g. 24. Let us recall the definition of a pseudomanifold with edges. Suppose that $M$ is a compact connected smooth manifold with boundary $\partial M$, and suppose that $\partial M$ is the total space of a smooth locally trivial bundle $\pi: \partial M \rightarrow N$ over a closed smooth base $N$ whose fiber is a closed smooth manifold. The topological space $X$ obtained by gluing $M$ to $N$ with help of $\pi$ (i.e. the points in each fiber $\pi^{-1}(s)$ are identified with $s \in N$ ) is called a pseudomanifold with edges corresponding to the pair $(M, \pi)$. The natural surjective map $M \rightarrow X$ which is the identity on $M \backslash \partial M$ is denoted by $\bar{\pi}$. In general, $N$ need not be connected, and the connected components of $N$ are called edges of $X$. Clearly, $X=(X \backslash N) \cup N$ is a decomposed space, moreover $X \backslash N$ is an open connected stratum of $X$. Now we will prove that $N$ satisfies the condition in Definition 2.2 For $s \in N$ let $L$ be the fiber $\pi^{-1}(s) \subset \partial M$ and $B$ be an open neighborhood of $s$ in $N$ such that $\pi^{-1}(B)=B \times \pi^{-1}(s)$. Let $\pi^{-1}(N)_{\varepsilon}$ be a collar neighborhood of the boundary component $\pi^{-1}(N) \subset \partial M$ in $M$ provided with a trivialization $(p, t): \pi^{-1}(N)_{\varepsilon} \rightarrow \pi^{-1}(N) \times[0, \varepsilon)$, where $p$ is a smooth retraction $\pi^{-1}(N)_{\varepsilon} \rightarrow \pi^{-1}(N)$. Set $U(s):=\bar{\pi}\left(p^{-1} \circ \pi^{-1}(B)\right)$. We define a trivialization $\phi_{s}: U(s) \rightarrow B \times c L(1)$ by $\phi_{s}(x)=(\pi \circ p(x),[t, p(x)])$. This shows that $X$ is a stratified space of depth 1 . An important class of pseudomanifolds with edges consists of pseudomanifolds $X$ with isolated conical singularities, if its edges $X_{i}$ of $X$ are just points $s_{i}$ of $X$.

2. If $L$ is a compact stratified space, then the cone $c L$ is also a stratified space.

3. If $L_{1}$ and $L_{2}$ are stratified spaces, then $L_{1} \times L_{2}$ is a stratified space. In particular, a product of cones $c L_{1} \times c L_{2}=c\left(L_{1} \times L_{2} \times[0,1]\right)$ has the following decomposition: $c\left(L_{1} \times L_{2} \times[0,1]\right)=\{p t\} \cup L_{1} \times(0,1) \cup L_{2} \times(0,1) \cup L_{1} \times L_{2} \times(0,1)$.

2.2. Smooth structure on stratified spaces and its simplest properties. We now introduce the notion of a smooth structure on a stratified space $X$, which is a natural extension of our notion of a smooth structure on a pseudomanifold with isolated conical singularities (pseudomanifold w.i.c.s.) in [16] (Remark 2.72).

As the notion of a stratified space $X$ is defined inductively on the depth of $X$, the notion of a smooth structure on $X$ is also defined inductively on the depth on $X$.

Since $X$ is locally modelled as a product $B \times c L(1)$, we define the notion of a smooth structure on the product $B \times c L(1)$ inductively on the depth of $L$. More generally, we introduce the notion of a smooth structure on a stratified space $X$ of depth $k$, recursively on $k$. The key notion is a product smooth structure (Definition 2.5.2).

For a stratum $S \subset X$ we consider the following associated spaces. 
- $C_{u}^{\infty}(S)$ - the space of all usual smooth functions on $S$.

- $C_{u, 0}^{\infty}(S):=C_{u}^{\infty}(S) \cap C_{0}^{0}(S)$, where $C_{0}^{0}(S)$ is the space of all continuous functions with compact support.

- For $f \in C_{0}^{0}(S)$, let $j_{*}(f) \in C_{0}^{0}(X)$ denote the unique extension of $f$ such that $j_{*}(f)=0$ if $x \notin S$.

Definition 2.5. (cf. 16, Definition 2.2]) A smooth structure on a stratified space $X$ of depth $k$ is a choice of a $\mathbb{R}$-subalgebra $C^{\infty}(X)$ of the algebra $C^{0}(X)$ of continuous real-valued functions on $X$ that satisfy the following properties.

1. $C^{\infty}(X)$ is a germ-defined $C^{\infty}$-ring.

2. For any $x \in X$ there exists a local trivialization $\phi_{x}: U(x) \rightarrow B_{x} \times c L(1)$ which is a local diffeomorphism of stratified spaces, i.e., $C^{\infty}(U)=\phi_{x}^{*}\left(C^{\infty}(B \times c L(1))\right)$, where $C^{\infty}(B \times c L(1))$ is a product smooth structure. In other words, $C^{\infty}(B \times$ $c L(1))$ is the germ-defined $C^{\infty}$-ring whose sheaf $S C^{\infty}(B \times c L(1))$ is generated by $\pi_{1}^{*}\left(S C^{\infty}(B)\right)$ and $\pi_{2}^{*}\left(S C^{\infty}(c L(1))\right)$, where $\pi_{1}$ and $\pi_{2}$ are the projections from $B \times c L(1)$ to $B$ and $c L(1)$ respectively (cf. [23, $\S 3]$ ).

3. A smooth structure $C^{\infty}(c L(1))$ on the cone over a compact stratified space $L$ must satisfy the following two additional properties: (3a) $\left.C^{\infty}(c L(1))\right|_{L \times(0,1)} \subset$ $C^{\infty}(L \times(0,1))$, and $(3 \mathrm{~b}) j_{*}\left[\pi_{1}^{*}\left(C_{u, 0}^{\infty}(0,1)\right)\right] \subset C^{\infty}\left(c(L(1))\right.$, where $\pi_{1}: L \times(0,1) \rightarrow$ $(0,1)$ is the projection.

Lemma 2.6. Any smooth structure $C^{\infty}(X)$ on a stratified space $X$ satisfies the following properties.

1. $C^{\infty}(X)_{\mid S} \subset C_{u}^{\infty}(S)$ for each stratum $S$ of $X$.

2. (cf. [16, Lemma 2.1]) $C^{\infty}(X)$ is partially invertible in the following sense. If $f \in C^{\infty}(X)$ is nowhere vanishing, then $1 / f \in C^{\infty}(X)$.

Proof. We prove the first assertion of Lemma 2.6 using induction on the dimension of $X$. Clearly this assertion is valid if $\operatorname{dim} X=1$. Since $C^{\infty}(X)$ is germ-defined, it suffices to prove Lemma 2.6.1 locally. Hence, w.l.o.g., by Definition 2.5 2 we can assume that $X=B \times c L(1)$ and $C^{\infty}(X)$ is a product smooth structure.

First, we consider the case $S=B \times[L, 0]$. By Definition 2.5.2, any smooth function on $X$ is locally written as $G\left(f_{1}, \cdots f_{n}, h_{1}, \cdots h_{k}\right)$ where $G \in C^{\infty}\left(\mathbb{R}^{n+k}\right)$, $f_{i} \in C^{\infty}(B), h_{i} \in C^{\infty}(c L(1))$. Since the restriction of $h_{i}$ to $B$ is constant, $G\left(f_{1}, \cdots, f_{n}, h_{1}, \cdots, h_{k}\right)_{\mid S}$ is a smooth function on $B$. Now assume that $S=$ $B \times S_{L} \times(0,1)$, where $S_{L}$ is a stratum of $L$. Using the condition (3a) in Definition 2.5, we can assume that $X=B \times L \times(0,1)$ and $C^{\infty}(X)$ is generated by $\pi_{1}^{*}\left(S C^{\infty}(B)\right), \pi_{2}^{*}\left(S C^{\infty}(L)\right), \pi_{3}^{*}\left(S C_{u}^{\infty}(0,1)\right)$, where $\pi_{1}, \pi_{2}, \pi_{3}$ are the projections from $X$ to $B, L,(0,1)$ respectively. Thus, any function in $C^{\infty}(X)$ is locally of the form $G\left(f_{i}, h_{j}, t\right)$ where $f_{i} \in C^{\infty}(B), h_{j} \in C^{\infty}(L)$ and $t \in(0,1)$. Using the induction assumption, noting that $\operatorname{dim} L \leq \operatorname{dim} X-1$, we have $\left.h_{i}\right|_{S_{L}} \in C_{u}^{\infty}\left(S_{L}\right)$. Hence $\left.G\right|_{B \times S_{L} \times(0,1)} \in C_{u}^{\infty}\left(B \times S_{L} \times(0,1)\right)$. This completes the proof of the first assertion of Lemma 2.6.

To prove the last assertion of Lemma 2.6 it suffices to show that locally $1 / f$ is a smooth function. Since $f \neq 0$, shrinking a neighborhood $U$ of $x$ if necessary, we can assume that there is an open interval $(-\varepsilon, \varepsilon)$ which has no intersection with $f(U)$. Now there exists a function $\psi: \mathbb{R} \rightarrow \mathbb{R}$ such that

a) $\left.\psi\right|_{f(U)}=I d$,

b) $(-\varepsilon / 2, \varepsilon / 2)$ does not intersect with $\psi(\mathbb{R})$. 
Clearly $G: \mathbb{R} \rightarrow \mathbb{R}$ defined by $G(x):=\psi(x)^{-1}$ is a smooth function. Note that $1 / f(y)=G(f(y))$ for any $y \in U$. This completes the proof of the last assertion of Lemma 2.6 .

Remark 2.7. 1. Denote by $i$ the canonical inclusion $X^{r e g} \rightarrow X$. Since $X=$ $\overline{X^{r e g}}$, the kernel of $i^{*}: C^{\infty}(X) \rightarrow C^{0}\left(X^{r e g}\right)$ is zero. Lemma 2.6.1 implies that $i^{*}\left(C^{\infty}(X)\right)$ is a subalgebra of $C_{u}^{\infty}\left(X^{r e g}\right)$. Roughly speaking, we can regard $C^{\infty}(X)$ as a subalgebra of $C_{u}^{\infty}\left(X^{r e g}\right)$.

2. The condition (3b) in Definition 2.5 is a relaxing of the condition 3 of Definition 2.2 in [16] for pseudomanifolds w.i.c.s. that requires $j_{*}\left(C_{0}^{\infty} M^{r e g}\right) \subset$ $C^{\infty}\left(M^{r e g}\right)$. In fact, in [16 (and in the present note) we need only the (weaker) condition (3b) of Definition 2.5 for the existence of partition of unity and nothing more.

3. Our definition of a smooth structure on a stratified space is a refinement of the definition due to Sjammar and Lerman [25], which requires a smooth structure to satisfy only Lemma 2.6.1. Pflaum introduces smooth structures by means of a maximal atlas; thus a smooth structure appears as an equivalence class of a system of local embeddings into suitable $\mathbb{R}^{n}[27$.

We are going to prove the existence of smooth partitions of unity, which is important for later applications (Remark 3.14, Remark 3.22).

Proposition 2.8. (cf. [16, Proposition 2.1]) Let $\left\{U_{i}\right\}_{i \in I}$ be a locally finite open covering of $X$ such that each $U_{i}$ has a compact closure $\bar{U}_{i}$. Then there exists a smooth partition of unity $\left\{f_{i}\right\}_{i \in I}$ subordinate to $\left\{U_{i}\right\}_{i \in I}$.

Proof. This is a local statement, hence it suffices to prove for the case $X=B \times$ $c L(1)$. Since the smooth structure on $B \times c L(1)$ is a product smooth structure, it is not hard to reduce Proposition 2.8 to the case $B$ is a point, i.e. $X=c L(1)$ is a cone over a stratified space $L$. For the case $L$ is a smooth manifold, by Remark 2.7.2, we have proved the corresponding assertion in [16. Proposition 2.1]. The proof of [16. Proposition 2.1] can be repeated word-by-word for the case $L$ is a stratified space, using the last two conditions of Definitions 2.5] so we omit its proof.

The following Corollary is an immediate consequence of the existence of partition of unity, see. e.g. [16, Lemma 2.11].

Corollary 2.9. Smooth functions on $X$ separate points on $X$.

Next, we introduce the notion of the cotangent bundle and the notion of the Zariski tangent bundle of a stratified space $X$, in the same way as we did in [16], which are similar to the notions introduced in [25], 27, B.1]. Note that the germs of smooth functions $C_{x}^{\infty}(X)$ is a local $\mathbb{R}$-algebra with the unique maximal ideal $\mathfrak{m}_{x}$ consisting of functions that vanish at $x$. Set $T_{x}^{*}(X):=\mathfrak{m}_{x} / \mathfrak{m}_{x}^{2}$. Since the following exact sequence

$$
0 \rightarrow \mathfrak{m}_{x} \rightarrow C_{x}^{\infty} \stackrel{j}{\rightarrow} \mathbb{R} \rightarrow 0
$$

splits, where $j$ is the evaluation map, $j\left(f_{x}\right)=f_{x}(x)$ for any $f_{x} \in C_{x}^{\infty}$, the space $T_{x}^{*} X$ can be identified with the space of Kähler differentials of $C_{x}^{\infty}(X)$. The Kähler derivation $d: C_{x}^{\infty}(X) \rightarrow T_{x}^{*} X$ is defined as follows:

$$
d\left(f_{x}\right):=\left(f_{x}-j^{-1}\left(f_{x}(x)\right)+\mathfrak{m}_{x}^{2},\right.
$$


where $j^{-1}: \mathbb{R} \rightarrow C_{x}^{\infty}$ is the left inverse of $j$, see e.g. [18, Chapter 10], or [27, Proposition B.1.2]. We call $T_{x}^{*} X$ the cotangent space of $X$ at $x$. Its dual space $T_{x}^{Z} X:=\operatorname{Hom}\left(T_{x}^{*} X, \mathbb{R}\right)$ is called the Zariski tangent space of $X$ at $x$. The union $T^{*} X:=\cup_{x \in X} T_{x}^{*} X$ is called the cotangent bundle of $X$. The union $T^{Z} X:=$ $\cup_{x \in X} T_{x}^{Z} X$ is called the Zariski tangent bundle of $X$.

Let us denote by $\Omega_{x}^{1}(X)$ the $C_{x}^{\infty}(X)$-module $C_{x}^{\infty}(X) \otimes_{\mathbb{R}} \mathfrak{m}_{x} / \mathfrak{m}_{x}^{2}$. We call $\Omega_{x}^{1}(X)$ the germs of 1-forms at $x$. Set $\Omega_{x}^{k}(X):=C_{x}^{\infty}(X) \otimes_{\mathbb{R}} \Lambda^{k}\left(\mathfrak{m}_{x} / \mathfrak{m}_{x}^{2}\right)$. Then $\oplus_{k} \Omega_{x}^{k}(X)$ is an exterior algebra with the following wedge product

$$
\left(f \otimes_{\mathbb{R}} d g_{1} \wedge \cdots \wedge d g_{k}\right) \wedge\left(f^{\prime} \otimes_{\mathbb{R}} d g_{k+1} \wedge \cdots \wedge d g_{l}\right):=\left(f \cdot f^{\prime}\right) \otimes_{\mathbb{R}} d g_{1} \wedge \cdots \wedge d g_{l},
$$

where $f, f^{\prime} \in C_{x}^{\infty}$ and $d g_{i} \in T_{x}^{*} M$.

Note that the Kähler derivation $d: C_{x}^{\infty}(X):=\Omega_{x}^{0}(X) \rightarrow \Omega_{x}^{1}(X)$ extends to the unique derivation $d: \Omega_{x}^{k}(X) \rightarrow \Omega_{x}^{k+1}(X)$ satisfying the Leibniz property. Namely we set

$$
\begin{array}{r}
d(f \otimes 1):=1 \otimes d f, \\
d(f \otimes \alpha \wedge g \otimes \beta):=d(f \otimes \alpha) \wedge g \otimes \beta+(-1)^{\operatorname{deg} \alpha} f \otimes \alpha \wedge d(g \otimes \beta) .
\end{array}
$$

Definition 2.10. 1. (cf. [23, §2]) A section $\alpha: X \rightarrow \Lambda^{k} T^{*}(X)$ is called a smooth differential $k$-form, if for each $x \in X$ there exists a neighborhood $U(x) \subset X$ of $x$ such that $\alpha(x)$ can be represented as $\sum_{i_{0} i_{1} \cdots i_{k}} f_{i_{0}} d f_{i_{1}} \wedge \cdots \wedge d f_{i_{k}}$ for some $f_{i_{0}}, \cdots, f_{i_{k}} \in C^{\infty}(X)$.

2. A section $V: X \rightarrow \Lambda^{k} T^{Z} X$ will be called a smooth Zariski $k$-vector field, if for any $\alpha \in \Omega^{k}(X)$ the value $V(\alpha)$ is a smooth function on $X$.

Denote by $\Omega(X)=\oplus_{k} \Omega^{k}(X)$ the space of all smooth differential forms on $X$. We identify the germ at $x$ of a $k$-form $\sum_{i_{0} i_{1} \cdots i_{k}} f_{i_{0}} d f_{i_{1}} \wedge \cdots \wedge d f_{i_{k}}$ with the element $\sum_{i_{0} i_{1} \cdots i_{k}} f_{i_{0}} \otimes d f_{i_{1}} \wedge \cdots \wedge d f_{i_{k}} \in \Omega_{x}^{k}(X)$. Clearly the Kähler derivation $d$ extends to a map, also denoted by $d$, that sends $\Omega(X)$ to $\Omega(X)$.

Now we set

Remark 2.7.1 implies immediately

$$
\Omega_{u}\left(X^{r e g}\right):=\Omega\left(X^{r e g}, C_{u}^{\infty}\left(X^{r e g}\right)\right) .
$$

Lemma 2.11. The kernel $i^{*}: \Omega(X) \rightarrow \Omega_{u}\left(X^{r e g}\right)$ is zero. Roughly speaking, we can regard $\Omega(X)$ as a subspace in $\Omega_{u}\left(X^{r e g}\right)$.

\subsection{Examples of smooth structures on stratified spaces.}

Example 2.12. Assume that $X$ is a realization of a compact polytope in $\mathbb{R}^{n}$, i.e., $X$ is a stratified space such that each stratum $S$ of dimension $k$ of $X$ is an open disk in some affine subspace of dimension $k$ in $\mathbb{R}^{n}$. Then $X$ has a natural smooth structure induced from the standard smooth structure on $\mathbb{R}^{n}$ i.e. $S C^{\infty}(X):=\left.S C^{\infty}\left(\mathbb{R}^{n}\right)\right|_{X}$. Indeed, by construction $C^{\infty}(X)$ is a germ-defined $C^{\infty}$ ring, hence the condition 1 in Definition 2.5 is trivially satisfied. Now let us prove the validity of the second condition on the product smooth structure inductively on the dimension of $X$. Note that the validity of the second condition is trivially satisfied, if $\operatorname{dim} X=0$. Since $X$ is a realization of a polytope, the tubular neighborhood of any point $x \in S^{k}$ in $\mathbb{R}^{n}$ has the form $B_{x} \times c L(1)$, where $L(1)$ is the intersection of $X$ with the sphere $S^{n-k-1}$ of a small enough radius centred at $x$ on a hyperplane through $x$ in $\mathbb{R}^{n}$ that is orthogonally complement to $S$, and $B_{x}$ is an open ball around $x$ in $S$. By the dimension induction assumption, $L(1) \subset S^{n-k-1}$ has a natural smooth structure induced from the embedding 
$L(1) \rightarrow S^{n-k-1} \subset \mathbb{R}^{n}$, which satisfies the conditions of Definition 2.5. since the projection from a punctured sphere $S^{n-k-1} \backslash\{p t\}$ to $\mathbb{R}^{n-k-1}$, where $\{p t\} \notin L(1)$, sends $L(1)$ to a realization of a polytope of lower dimension in $\mathbb{R}^{n-k-1}$, and this projection is a diffeomorphism between $S^{n-k-1} \backslash\{p t\}$ and $\mathbb{R}^{n-k-1}$. To study the smooth structure on $c L(1)$ we need the following

Lemma 2.13. Assume that $A \subset \mathbb{R}^{n}$ and $B \subset \mathbb{R}^{m}$. Then the sheaf $S C^{\infty}\left(\mathbb{R}^{n} \times\right.$ $\left.\mathbb{R}^{m}\right)\left.\right|_{A \times B}$ is generated by $\pi_{1}^{*}\left(\left.S C^{\infty}\left(\mathbb{R}^{n}\right)\right|_{A}\right)$ and $\pi_{2}^{*}\left(\left.S C^{\infty}\left(\mathbb{R}^{m}\right)\right|_{B}\right)$, where $\pi_{1}$ and $\pi_{2}$ are the projection of $\mathbb{R}^{n+m}$ onto $\mathbb{R}^{n}$ and $\mathbb{R}^{m}$ respectively.

Proof. Lemma 2.13 is a consequence of the simple fact that $S C^{\infty}\left(\mathbb{R}^{n} \times \mathbb{R}^{m}\right)$ is generated by $S C^{\infty}\left(\mathbb{R}^{n}\right)$ and $S C^{\infty}\left(\mathbb{R}^{m}\right)$.

Lemma 2.13 implies that the induced smooth structure on the product $B_{x} \times$ $c L(1)$ satisfies the condition 2 of Definition 2.5. The condition (3a) of Definition 2.5 trivially holds, and the condition (3b) of Definition 2.5 also holds for the smooth structure on the cone $c L(1) \subset \mathbb{R}^{n-k} \subset \mathbb{R}^{n}$, using partition of unity on $\mathbb{R}^{n-k}$. This proves that the induced smooth structure on $X$ satisfies all the conditions of Definition 2.5

Example 2.14. Assume that $\left(X_{i}, C^{\infty}\left(X_{i}\right)\right)$ are stratified spaces provided with smooth structures. Then it is easy to verify that $\left(\Pi X_{i}, \Pi\left(C^{\infty}\left(X_{i}\right)\right)\right.$ is a stratified manifold provided with a smooth structure.

Example 2.15. Assume that we have a continuous surjective map $M \stackrel{\pi}{\rightarrow} X$ from a smooth manifold $M$ with corner to a stratified space $X$ of depth 1 such that for each stratum $S_{i} \subset X$ the triple $\left(\pi^{-1}\left(S_{i}\right), \pi_{i}, S_{i}\right)$ is a differentiable fibration, moreover for each $x \in X^{\text {reg }}$ the preimage $\pi^{-1}(x)$ consists of a single point. Clearly $\pi$ induces a stratified space structure on $M$. The $\mathbb{R}$-subalgebra $C^{\infty}(X):=\{f \in$ $\left.C^{0}(X) \mid \pi^{*} f \in C^{\infty}(M)\right\}$ will be called a resolvable smooth structure. We are going to show that a resolvable smooth structure satisfies the conditions in Definition 2.5. First, $C^{\infty}(X)$ is a germ-defined $C^{\infty}$-ring, since $C^{\infty}(M)$ possesses this property. Next, the existence of a local smooth trivialization $\phi_{x}$ for each $x \in X$, which satisfies the last two conditions of Definition 2.5 is a consequence of the existence of a differentiable fibration $\left(\pi^{-1}\left(S_{i}\right), \pi_{i}, S_{i}\right)$ and the fact that $\pi$ induces a stratified space structure on $M$. The space $M$ will be called a resolution of $X$.

In what follows we study some properties of a resolvable smooth structure on a stratified space of depth 1 .

We say that $C^{\infty}(M)$ is locally smoothly contractible, if for any $x \in M$ there exists an open neighborhood $U(x) \ni x$ together with a smooth homotopy $\sigma$ : $U(x) \times[0,1] \rightarrow U(x)$ joining the identity map with the constant map $U(x) \mapsto x$ [23, §5].

A $C^{\infty}$-ring $C^{\infty}(X)$ is called finitely generated, if there are finite elements $f_{1}, \cdots, f_{k} \in C^{\infty}(X)$ such that any $h \in C^{\infty}(X)$ can be written as $h=G\left(f_{1}, \cdots, f_{k}\right)$, where $G \in C^{\infty}\left(\mathbb{R}^{k}\right)$.

Lemma 2.16. Every pseudomanifold $X$ with edges has a resolvable smooth structure, which is locally smoothly contractible.

Proof. By definition (see Example 2.4.1), there exist a compact smooth manifold $M$ with boundary $\partial M$ and a surjective map $\bar{\pi}: M \rightarrow X$. In Example 2.12.3 we have shown that such a $X$ has a resolvable smooth structure $C^{\infty}(X):=\{f \in$ 
$\left.C^{0}(X) \mid \bar{\pi}^{*} f \in C^{\infty}(M)\right\}$. We will show that $C^{\infty}(X)$ is locally smoothly contractible. Let $S_{i}$ be a singular stratum of $X$, and $\bar{\pi}^{-1}\left(S_{i}\right)=\partial M_{i} \subset \partial M$. Let $V\left(\partial M_{i}\right)$ be a collar open neighborhood of $\partial M_{i}$ in $M$. Then $U\left(S_{i}\right):=\bar{\pi}\left(V\left(\partial M_{i}\right)\right)$ is an open neighborhood of $S_{i}$ in $X$. Let us consider the following commutative diagram

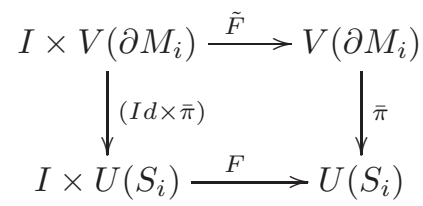

where $\tilde{F}$ is a smooth retraction from $V\left(\partial M_{i}\right)$ to $\partial M_{i}$, constructed using the fibration $[0,1) \rightarrow V\left(\partial M_{i}\right) \rightarrow \partial M_{i}$. We set

$$
F(t, x):=\bar{\pi}\left(\tilde{F}\left(t, \bar{\pi}^{-1}(x)\right)\right) .
$$

Since $\tilde{F}_{\mid \partial M_{i}}=I d$, the map $F$ is well-defined. Clearly $F$ is a smooth homotopy, since $\tilde{F}$ is a smooth homotopy. This proves Proposition 2.16 .

Proposition 2.17. A resolvable smooth structure on a stratified space $X$ of depth 1 obtained from a smooth manifold $M$ with corner is not finitely generated as a $C^{\infty}$-ring, if there exists $x \in X$ such that $\operatorname{dim} \pi^{-1}(x) \geq 1$, where $\pi: M \rightarrow X$ is the associated projection.

Proof. Assume the opposite i.e. $C^{\infty}(X)$ is generated by $g_{1}, \cdots, g_{n} \in C^{\infty}(X)$. Then $G:=\left(g_{1}, \cdots, g_{n}\right)$ defines a smooth embedding $X \rightarrow \mathbb{R}^{n}$. Hence $C^{\infty}(X)=$ $C^{\infty}\left(\mathbb{R}^{n}\right) / I$, where $I$ is an ideal of $C^{\infty}\left(\mathbb{R}^{n}\right)$ of smooth functions on $\mathbb{R}^{n}$ vanishing on $G(X)$ [21, p. 21, Proposition 1.5]. In particular, the cotangent space $T_{x}^{*} X$ is a finite dimensional linear space for all $x \in X$. We will show that this assertion leads to a contradiction.

Let $S$ be a stratum of $X$ such that $\operatorname{dim}\left(\pi^{-1}(S)\right) \geq \operatorname{dim} S+1$. Let $x \in S$ and $U(x)$ a small open neighborhood of $x$ in $X$. Let $f \in C^{\infty}(U(x))$, equivalently $\pi^{*}(f) \in$ $C^{\infty}\left(\pi^{-1}(U(x))\right.$. Let $\chi: \pi^{-1}(U(x)) \rightarrow \mathbb{R}_{+}^{p} \times \mathbb{R}^{n-p} \subset \mathbb{R}^{n}$ be a coordinate map on $\pi^{-1}(U(x)) \subset M$. By definition of manifolds with corner, we have $\left(\chi^{-1}\right)^{*} \pi^{*}(f) \in$ $C^{\infty}(\tilde{U})$ for some open set $\tilde{U} \subset \mathbb{R}^{n}$ containing $\chi\left(\pi^{-1}(U(x))\right)$. Denote by $\tilde{S}$ the preimage $\chi \circ \pi^{-1}(S \cap U(x))$, which is a submanifold of $\tilde{U}(x)$. Let us denote the restriction of $\pi \circ \chi^{-1}$ to $\tilde{S}$ by $\tilde{\pi}$. Then the triple $(\tilde{S}, \tilde{\pi}, S \cap U)$ is a smooth fibration, whose fiber $\tilde{\pi}^{-1}(y)$ is a smooth manifold of dimension at least 1 . We note that $\left(\chi^{-1}\right)^{*} \pi^{*}(f)$ belongs to the subalgebra $C^{\infty}(\tilde{U}, \tilde{S}, \tilde{\pi})$ consisting of smooth functions on $\tilde{U}$ that are constant along fiber $\tilde{\pi}^{-1}(y)$ for all $y \in S \cap U(x)$. Since the depth of $X$ is $1, C^{\infty}(\tilde{U}, \tilde{S}, \tilde{\pi})$ is identified with the set of smooth functions on $U$.

Shrinking $U(x)$ we can assume that $\tilde{S}=\tilde{U} \cap \mathbb{R}^{k}$ and $\tilde{\pi}: \tilde{S} \rightarrow S \cap U$ is the restriction of a linear projection $\bar{\pi}: \mathbb{R}^{k} \rightarrow \mathbb{R}^{l}$, where $l=\operatorname{dim} S, k=\operatorname{dim} \tilde{S}$, and $S \cap U=\mathbb{R}^{l} \cap U$. Here we assume that $U$ is an open set in $\mathbb{R}^{n}$. Let $\mathbb{R}^{n-k}$ with coordinate $\tilde{x}=\left(\tilde{x}^{1}, \cdots, \tilde{x}^{n-k}\right)$ be a complement to $\mathbb{R}^{k}$ in $\mathbb{R}^{n} \supset \tilde{U}$, and let $\mathbb{R}^{k-l} \subset \mathbb{R}^{k}$ with coordinate $\tilde{y}=\left(\tilde{y}^{1}, \cdots, \tilde{y}^{k-l}\right)$ be the set $\bar{\pi}^{-1}(0)$. We also equip the subspace $\mathbb{R}^{l}$ with coordinate $\tilde{z}=\left(\tilde{z}^{1}, \cdots, \tilde{z}^{l}\right)$. The condition $\operatorname{dim} \pi^{-1}(x) \geq 1$ in Proposition 2.16 is equivalent to the equality $k-l \geq 1$; in other words, $y$ is an essential variable. Furthermore, a point $\tilde{s} \in \tilde{S} \subset \mathbb{R}^{n}$ has (local) coordinates with $\tilde{x}=0$. 
Lemma 2.18. A function $g \in C^{\infty}(\tilde{U})$ belongs to $C^{\infty}(\tilde{U}, \tilde{S}, \tilde{\pi})$ if and only if $g$ has the form

$$
g\left(\tilde{x}^{1}, \cdots, \tilde{x}^{n-k}, \tilde{y}, \tilde{z}\right)=\tilde{x}^{1} g_{1}(\tilde{x}, \tilde{y}, \tilde{z})+\cdots+\tilde{x}^{n-k} g_{n-k}(\tilde{x}, \tilde{y}, \tilde{z})+c(\tilde{z}),
$$

where $g_{i} \in C^{\infty}(\tilde{U})$ and $c(\tilde{z})$ is a smooth function on $U$ depending only on variable $\tilde{z}$.

Proof. We write for $g \in C^{\infty}(\tilde{U}, \tilde{S}, \tilde{\pi})$

$$
g(\tilde{x}, \tilde{y}, \tilde{z})-g(0, \tilde{y}, \tilde{z})=\int_{0}^{1} \frac{d g(t \tilde{x}, \tilde{y}, \tilde{z})}{d t} d t=\int_{0}^{1} \sum_{i=1}^{n-k} \frac{\partial g\left(t \tilde{x}^{1}, \cdots, t \tilde{x}^{n-k}, \tilde{y}, \tilde{z}\right)}{\partial\left(t \tilde{x}^{i}\right)} \tilde{x}_{i} d t .
$$

Setting

$$
g_{i}:=\int_{0}^{1} \frac{\partial g\left(t \tilde{x}^{1}, \cdots, t \tilde{x}^{n-k}, \tilde{y}, \tilde{z}\right)}{\partial\left(t \tilde{x}^{i}\right)} d t
$$

we obtain $g(\tilde{x}, \tilde{y}, \tilde{z})=\sum_{i=1}^{n-k} \tilde{x}^{i} g_{i}(\tilde{x}, \tilde{y}, \tilde{z})+g(0, \tilde{y}, \tilde{z})$. Since $g(0, \tilde{y}, \tilde{z})$ depends only on $\tilde{z}$, we obtain the "only if" part of Lemma 2.18 immediately. The "if" part is trivial. This proves Lemma 2.18 .

Now let us complete the proof of Proposition 2.17. Take a point $s \in S$ and a point $\tilde{s} \in \tilde{\pi}^{-1}(s)$ such that $\tilde{x}(\tilde{s})=\tilde{y}(\tilde{s})=\tilde{z}(\tilde{s})=0$. Since $X$ has depth 1, Lemma 2.18 implies that the maximal ideal $\mathfrak{m}_{s}$ is a linear space generated by functions of the form $\tilde{x}^{i} g_{i, \alpha}(\tilde{x}, \tilde{y}, \tilde{z}), i=\overline{1, n-k}$. Let us consider the sequence $S:=$ $\left\{\tilde{x}^{1} \tilde{y}^{1}, \cdots, \tilde{x}^{1}\left(\tilde{y}^{1}\right)^{m} \in \mathfrak{m}_{s}\right\}, m \rightarrow \infty$. If $\operatorname{dim} T_{s}^{*} X=\operatorname{dim} \mathfrak{m}_{s} / \mathfrak{m}_{s}^{2}=n$, there exists a subsequence $\tilde{x}^{1}\left(\tilde{y}^{1}\right)^{k_{1}}, \cdots, \tilde{x}^{1}\left(\tilde{y}^{1}\right)^{k_{n}}$ such that $\tilde{x}^{1}\left(\tilde{y}^{1}\right)^{m}$ is a linear combination of $\tilde{x}^{1}\left(\tilde{y}^{1}\right)^{k_{j}}$ modulo $\mathfrak{m}_{s}^{2}$ for any $m$, which is impossible. This completes the proof of Proposition 2.17.

Remark 2.19. Proposition 2.17 partially answers the question 2 we posed in [16, $\S 5]$. We observe that there are many quotient smooth structures which are finitely generated, i.e. a quotient by a smooth group action. In this case the dimension of the fiber over singular strata (e.g. the dimension of a singular orbit) is smaller than or equal to the dimension of the generic fiber (the dimension of a generic orbit, respectively).

\section{Symplectic Stratified SpaCes AND COMPATible SMooth StRuCtures}

In this chapter we introduce the notion of a stratified symplectic space $(X, \omega)$ (Definition 3.1), which is close to that introduced by Sjamaar and Lerman 25] (Remark 3.2). We also introduce the notion of a weakly symplectic smooth structure and the notion of a Poisson smooth structure on $(X, \omega)$ (Definition 3.3). We give examples of weakly symplectic smooth structures and Poisson smooth structures (Propositions 3.8, 3.11, Examples 3.9.1-3). We prove the existence and uniqueness of a Hamiltonian flow associated with a smooth function $H$ on a symplectic stratified space $X$, which is equipped with a Poisson smooth structure (Theorem [3.13). We compare our result with a result by Sjamaar and Lerman in [25, §3] (Remark 3.14). We prove that the Brylinski-Poisson homology of a symplectic stratified space $X$ provided with a Poisson weakly symplectic smooth structure is isomorphic to the de Rham cohomology of $X$, if the regular strata of $X$ have the same dimension (Theorem 3.16) Then we show that, under a mild condition, a stratified symplectic space $(X, \omega)$ provided with a Poisson weakly symplectic 
smooth structure $C^{\infty}(X)$ enjoys many nice properties related to the existence of a Lefschetz decomposition (Lemma 3.18, Proposition 3.20, Theorem 3.21).

\subsection{Symplectic stratified spaces.}

Definition 3.1. A stratified space $X$ is called symplectic, if every stratum $S_{i}$ is provided with a symplectic form $\omega_{i}$. The collection $\omega:=\left\{\omega_{i}\right\}$ is called a stratified symplectic form, or simply a symplectic form, if no misunderstanding can occur.

Remark 3.2. Definition 3.1 coincides with the first topological condition in 25 , Definition 1.12.(i)] of a symplectic stratified space $X$ introduced by Sjamaar and Lerman. (The other conditions [25, Definitions 1.12.(ii), 1.12.(iii)] require the existence of a compatible smooth structure on $X$, which is also called by other authors 13 a stratified symplectic Poisson algebra). Thus any stratified symplectic space in Sjamaar's and Lerman's definition is a stratified symplectic space in our definition.

3.2. Weakly symplectic smooth structures and Poisson smooth structures. On each symplectic stratum $\left(S_{i}, \omega_{i}\right)$ we define the bivector $G_{\omega_{i}}$ to be the section of the bundle $\Lambda^{2} T S_{i}$ such that $G_{\omega_{i}}(x)=\partial y_{1} \wedge \partial x_{1}+\cdots+\partial y_{n} \wedge \partial x_{n}$ if $\omega_{i}(x)=\sum_{j=1}^{n} d x^{j} \wedge d y^{j}$ [3, §1.1]. If we regard $\omega_{i}$ as an element in $\operatorname{End}\left(T S_{i}, T^{*} S_{i}\right)$ and $G_{\omega_{i}}$ as an element in $\operatorname{End}\left(T^{*} S_{i}, T S_{i}\right)$, then $G_{\omega_{i}}$ is the inverse of $\omega_{i}$. The bivector $G_{\omega_{i}}$ defines a Poisson bracket on $C^{\infty}\left(S_{i}\right)$ by setting $\{f, g\}_{\omega_{i}}:=G_{\omega_{i}}(d f \wedge d g)$.

Definition 3.3. Let $(X, \omega)$ be a symplectic stratified space and $C^{\infty}(X)$ be a smooth structure on $X$.

1. A smooth structure $C^{\infty}(X)$ is said to be weakly symplectic, if there is a smooth 2-form $\tilde{\omega} \in \Omega^{2}(X)$ such that the restriction of $\tilde{\omega}$ to each stratum $S_{i}$ coincides with $\omega_{i}$. In this case we also say that $\tilde{\omega}$ is compatible with $C^{\infty}(X)$.

2. A smooth structure $C^{\infty}(X)$ is called Poisson, if there is a Poisson structure $\{,\}_{\omega}$ on $C^{\infty}(X)$ such that $\left(\{f, g\}_{\omega}\right)_{\mid S_{i}}=\left\{f_{\mid S_{i}}, g_{\mid S_{i}}\right\}_{\omega_{i}}$ for any stratum $S_{i} \subset X$.

Remark 3.4. 1. Lemma 2.11 implies that there exists at most one 2 -form $\tilde{\omega} \in$ $\Omega^{2}(X)$ which is compatible with a given smooth structure $C^{\infty}(X)$.

2. We claim that the condition 2 in Definition 3.3 is equivalent to the existence of a smooth Zariski bi-vector field $\tilde{G}_{\omega} \in \Gamma\left(\Lambda^{2} T^{Z}(X)\right)$ such that

$$
\tilde{G}_{\omega}(\alpha)_{\mid S_{i}}=G_{\omega_{i}}\left(\alpha_{\mid S_{i}}\right)
$$

for any stratum $S_{i} \subset X$. Indeed, the existence a section $\tilde{G}_{\omega}$ satisfying (3.1) defines a Poisson structure on $C^{\infty}(X)$ by setting $\{f, g\}(x):=\tilde{G}_{\omega}(d f \wedge d g)(x)$. Conversely, assume that there is a Poisson structure $\{,\}_{\omega}$ on $C^{\infty}(X)$ whose restriction to each stratum $S_{i}$ coincides with the given Poisson structure on $S_{i}$. We claim the bivector $\tilde{G}_{\omega_{i}}$ is a smooth Zariski bi-vector field. Since the space of smooth differential forms is germ-defined, it suffices to show the above claim locally. Note that on some neighborhood $U$ we can write $\Omega^{2}(X) \ni \alpha=\sum_{i} f_{i} d g_{i} \wedge d h_{i}$, where $f_{i}, g_{i}, h_{i} \in$ $C^{\infty}(U)$. Since the smooth structure is Poisson, we get

$$
\tilde{G}_{\omega}(\alpha)=\sum_{i} \tilde{G}_{\omega}\left(f_{i} d g_{i} \wedge d h_{i}\right)=\sum_{i} f_{i}\left\{g_{i}, h_{i}\right\} \in C^{\infty}(U) .
$$

This proves our claim.

3. The condition 2 of Definition 3.3 agrees with the condition (iii) in Definition 1.12 of 25 by Sjamaar and Lerman of a stratified symplectic Poisson algebra. 
It also agrees with our Definition of a Poisson smooth structure on a conical symplectic pseudomanifold in [16, §4].

Now we are going to consider important examples of weakly symplectic smooth structures and Poisson smooth structures.

Example 3.5. We assume that a compact Lie group $G$ acts on a connected symplectic manifold $(M, \omega)$ with proper moment map $J: M \rightarrow \mathfrak{g}^{*}$. Let $Z=$ $J^{-1}(0)$. The quotient space $M_{0}=Z / G$ is called a symplectic reduction of $M$. If 0 is a singular value of $J$ then $Z$ is not a manifold and $M_{0}$ is called a singular symplectic reduction. It is known that $M_{0}$ is a stratified symplectic space in Sjamaar's and Lerman's definition [25], and hence in our definition, see Remark 3.2. Let us recall the description of $M_{0}$ by Sjamaar and Lerman. For a subgroup $H$ of $G$ denote by $M_{(H)}$ the set of all points whose stabilizer is conjugate to $\mathrm{H}$, the stratum of $M$ of orbit type $(H)$.

Lemma 3.6. 25, Theorem 2.1] Let $(M, \omega)$ be a Hamiltonian $G$-space with moment map $J: M \rightarrow \mathfrak{g}^{*}$. The intersection of the stratum $M_{(H)}$ of orbit type $(H)$ with the zero level set $Z$ of the moment map is a manifold, and the orbit space

$$
\left(M_{0}\right)_{(H)}=\left(M_{(H)} \cap Z\right) / G
$$

has a natural symplectic structure $\left(\omega_{0}\right)_{(H)}$ whose pullback to $Z_{(H)}:=M_{(H)} \cap Z$ coincides with the restriction to $Z_{(H)}$ of the symplectic form $\omega$ on $M$. Consequently the stratification of $M$ by orbit types induces a decomposition of the reduced space $M_{0}=Z / G$ into a disjoint union of symplectic manifolds $M_{0}=\cup_{H \subset G}\left(M_{0}\right)_{(H)}$.

Since $J$ is proper, by Theorem 5.9 in [25] the regular part $M_{0}^{r e g}$ is connected. Sjamaar and Lerman also defined a "canonical" smooth structure on $M_{0}$ as follows. Set $C^{\infty}\left(M_{0}\right)_{\text {can }}:=C^{\infty}(M)^{G} / I^{G}$, where $I^{G}$ is the ideal of $G$-invariant functions vanishing on $Z$ [25, Example 1.11]. We will show that $C^{\infty}\left(M_{0}\right)_{\text {can }}$ is also a smooth structure in the sense of Definition 2.5. Denote by $\pi$ the natural projection $Z \rightarrow$ $Z / G$. Set $C^{\infty}(Z):=\left.C^{\infty}(M)\right|_{Z}$. Since $Z$ is closed, $C^{\infty}(Z)=C^{\infty}(M) / I_{Z}$, where $I_{Z}$ is the ideal of smooth functions on $M$ vanishing on $Z$.

We claim that the space $C^{\infty}(Z)^{G}$ of $G$-invariant smooth functions on $Z$ can be identified with the space $C^{\infty}\left(M_{0}\right)_{c a n}=C^{\infty}(M)^{G} / I^{G}$. Clearly $C^{\infty}(M)^{G} / I^{G}$ is a subspace of $G$-invariant smooth functions on $Z$. On the other hand, any smooth function $f$ on $M$ can be modified to a $G$-invariant smooth function $f_{G} \in C^{\infty}(M)$ by setting

$$
f_{G}(x):=\int_{G} f(g \cdot x) \mu_{g}
$$

for a $G$-invariant measure $\mu_{g}$ on $G$ normalized by the condition $\operatorname{vol}(G)=1$. So if $g \in C^{\infty}(Z)^{G}$, then $g$ is the restriction of a $G$-invariant function on $M$. In other words, we have an injective map $C^{\infty}(Z)^{G} \rightarrow C^{\infty}(M)^{G} / I^{G}$. Hence follows the identity $C^{\infty}(Z)^{G}=C^{\infty}\left(M_{0}\right)_{c a n}$. It follows that $C^{\infty}\left(M_{0}\right)_{c a n}$ is the quotient of the smooth structure obtained from $C^{\infty}(Z)$ via the projection $\pi: Z \rightarrow M_{0}$. In particular, $C^{\infty}\left(M_{0}\right)_{\text {can }}$ is a germ-defined $C^{\infty}$-ring, since $C^{\infty}(Z)$ is a germ-defined $C^{\infty}$-ring.

Proposition 3.7. $C^{\infty}\left(M_{0}\right)_{c a n}$ is a smooth structure in the sense of Definition 2.5. 
Proof. Note that $C^{\infty}\left(M_{0}\right)_{\text {can }}$ satisfies the first condition in Definition2.5. To show that $C^{\infty}\left(M_{0}\right)_{\text {can }}$ satisfies the other conditions in Definition 2.5, we use Theorem 6.7 in $[25]$ which asserts that there is a proper smooth embedding $\left(M_{0}, C^{\infty}\left(M_{0}\right)_{\text {can }}\right) \stackrel{i}{\rightarrow}$ $\left(\mathbb{R}^{n}, C^{\infty}\left(\mathbb{R}^{n}\right)\right)$ such that the image $i\left(M_{0}\right)$ is a stratified Whitney subspace $X$ of $\mathbb{R}^{n}$. In particular $C^{\infty}\left(M_{0}\right)_{\text {can }}=i^{*}\left(C^{\infty}\left(\mathbb{R}^{n}\right)\right)$.

We describe a neighborhood of a point $p \in X$ in $\mathbb{R}^{n}$ following [25, p. 410]. Let $S$ denote the stratum of $X$ that contains $p$. Let $N^{\prime}$ be a submanifold in $\mathbb{R}^{n}$ that is transversal to each stratum of $X$, intersects $S$ in the single point $p$ and satisfies $\operatorname{dim} N^{\prime}+\operatorname{dim} S=n$. Let $B_{\delta}(p) \subset \mathbb{R}^{n}$ denote the ball of radius $\delta$. By Whitney's condition $\mathrm{B}$, if $\delta$ is sufficiently small, then the sphere $\partial B_{\delta}(p)$ will be transversal to to each stratum in $X \cap N^{\prime}$. Fix such a $\delta>0$. Next we consider the normal slice $N(p):=N^{\prime} \cap X \cap B_{\delta}(p)$ and the link $L(p):=N^{\prime} \cap X \cap \partial B_{\delta}(p)$ of the stratum $S$ at the point $p$. These spaces are canonical Whitney stratified spaces, since they are transversal intersections of Whitney stratified spaces. Furthermore, $S$ has an open neighborhood $T_{S}$ in $X$ with local trivial fibration $\pi: T_{S} \rightarrow S$ such that $\pi^{-1}(p)$ is homeomorphic to $c L(p)$. Using Lemma 2.13 this implies that the induced smooth structure on $X$ satisfies the condition 2 of Definition 2.5. The last two conditions (3a) and (3b) of Definition 2.5 also hold for $X$, since locally we have the same description of $X$ as that in Example 2.12. This completes the proof of Proposition 3.7. (We also refer the reader to a more explicit, algebraic description of the local structure of $C^{\infty}\left(M_{0}\right)_{c a n}$ given in Theorem 5.1 [25] and its proof).

Proposition 3.8. The smooth structure $C^{\infty}\left(M_{0}\right)_{\text {can }}$ is both weakly symplectic and Poisson.

Proof. We observe that $C^{\infty}\left(M_{0}\right)_{\text {can }}$ is weakly symplectic, since by Proposition 3.6 the pull back $\pi^{*}\left(\omega_{0}\right)$ is equal to the restriction of the symplectic form $\omega$ to $Z$. Furthermore, the Poisson property of $C^{\infty}\left(M_{0}\right)_{\text {can }}$ follows from 25, Proposition 3.1], where they showed that $C^{\infty}\left(M_{0}\right)_{\text {can }}$ is closed under the Poisson bracket. This completes the proof of Proposition 3.8

Let us consider another important class of stratified symplectic spaces, which are the closure of nilpotent orbits in a complex semisimple Lie algebra $\mathfrak{g}$. This class has been examined by Panyshev [26, Huebschmann [13, Fu 8] and many other under different perspectives.

Example 3.9. For $x \in \mathfrak{g}$ let $x=x_{s}+x_{n}$ be the Jordan decomposition of $x$, where $x_{n} \neq 0$ is a nilpotent element, $x_{s}$ is a semisimple and $\left[x_{s}, x_{n}\right]=0$. Denote by $G$ the adjoint group of $\mathfrak{g}$ and by $\mathcal{Z}_{G}\left(x_{s}\right)$ the centralizer of $x_{s}$ in $G$. The adjoint orbit $G(x)$ is a fibration over $G\left(x_{s}\right)$ whose fiber over $x_{s}$ is the $\mathcal{Z}_{G}\left(x_{s}\right)$-orbit of $x_{n}$. Since $G\left(x_{s}\right)$ is a closed orbit, a neighborhood $U$ of a point $x \in \overline{G(x)}$ is isomorphic to the product $B \times \overline{\mathcal{Z}_{G}}\left(x_{s}\right) \cdot x_{n}$, where $B$ is an open neighborhood of $x_{s}$ in $G\left(x_{s}\right)$. It is known that the closure $\overline{\mathcal{Z}_{G}\left(x_{s}\right) \cdot x_{n}}$ is a finite union of $\mathcal{Z}_{G}\left(x_{s}\right)$-orbits of nilpotent elements in the Lie subalgebra $\mathcal{Z}_{\mathfrak{g}}\left(x_{s}\right)$ [6, chapter 6], so the closure $\overline{G(x)}$ is a finite union of adjoint orbits in $\mathfrak{g}$ provided with the Kostant-Kirillov symplectic structure. Thus $\overline{G(x)}$ is a decomposed space, whose strata are symplectic manifolds. Moreover $\overline{G(x)}^{r e g}=G(x)$ is connected.

1. Now assume that $x_{n}$ is a minimal nilpotent element in $\mathcal{Z}_{\mathfrak{g}}\left(x_{s}\right)$. Then $\overline{G(x)}$ is a stratified symplectic space of depth 1 , since $\overline{\mathcal{Z}_{G}\left(x_{s}\right) \cdot x_{n}}=\mathcal{Z}_{G}\left(x_{s}\right) \cdot x_{n} \cup\{0\}$, 6 , 
$\S 4.3]$. The embedding $\overline{G(x)} \rightarrow \mathfrak{g}$ provides $\overline{G(x)}$ with a natural finitely generated $C^{\infty}$-ring

$$
C_{1}^{\infty}(\overline{G(x)}):=\left\{f \in C^{0}(\overline{G(x)}) \mid f=\tilde{f}_{\mid \overline{G(x)}} \text { for some } \tilde{f} \in C^{\infty}(\mathfrak{g})\right\} .
$$

Clearly, $C_{1}^{\infty}(\overline{G(x)})$ satisfies the first condition of Definition 2.5 The last two conditions in Definition 2.5 also hold, since $\overline{G(x)}$ is a fibration over $G\left(x_{s}\right)$ whose fiber is the cone $\overline{\mathcal{Z}_{G\left(x_{s}\right)}\left(x_{n}\right)}$ containing the origin $\{0\} \in \mathfrak{g}$. Thus $C_{1}^{\infty}(\overline{G(x)})$ is a smooth structure according to Definition 2.5 .

The smooth structure $C_{1}^{\infty}(\overline{G(x)})$ is Poisson that is inherited from the Poisson structure on $\mathfrak{g}$. It is also weakly symplectic, since the symplectic form on $\overline{G(x)}$ is the restriction of the smooth 2 -form $\omega_{x}(v, w)=\langle x,[v, w]\rangle$ on $\mathfrak{g}$.

2. We still assume that $x_{n}$ is minimal in $\mathcal{Z}_{\mathfrak{g}}\left(x_{s}\right)$. In [26, Lemma 2] Panyushev showed that $\overline{G(x)}$ possesses an algebraic (Springer's) resolution of the singularity at $\{0\} \in \overline{G(x)} \subset \mathfrak{g}$. We will show that this resolution brings a resolvable smooth structure $C_{2}^{\infty}(\overline{G(x)})$. First we recall the construction in [26]. Let

- $h$ be a characteristic of $x_{n}$ (i.e. $h \in \mathcal{Z}_{\mathfrak{g}}\left(x_{s}\right)$ is a semisimple element and $\left(h, x_{n}, y_{n}\right) \subset \mathcal{Z}_{\mathfrak{g}}\left(x_{s}\right)$ is an $\mathfrak{s} l_{2}$-triple);

- $\mathcal{Z}_{\mathfrak{g}}\left(x_{s}\right)(i):=\left\{s \in \mathcal{Z}_{\mathfrak{g}}\left(x_{s}\right) \mid[h, s]=i s\right\}$

- $\mathfrak{n}_{2}\left(x_{s}\right):=\oplus_{i \geq 2} \mathcal{Z}_{\mathfrak{g}}\left(x_{s}\right)(i)$

- $P\left(x_{s}\right)$ denote a parabolic subgroup with the Lie algebra $l P\left(x_{s}\right):=\oplus_{i \geq 0} \mathcal{Z}_{\mathfrak{g}}\left(x_{s}\right)(i)$

- $N_{-}$- the connected Lie subgroup of $\mathcal{Z}_{G}\left(x_{s}\right)$ with Lie algebra

$l N_{-}\left(x_{s}\right):=\oplus_{i<0} \mathcal{Z}_{\mathfrak{g}}\left(x_{s}\right)(i)$.

It is known that $\overline{P\left(x_{s}\right) \cdot x_{n}}=\mathfrak{n}_{2}\left(x_{s}\right)$ and $\mathcal{Z}_{\mathcal{Z}_{G\left(x_{s}\right)}}\left(x_{n}\right) \subset P\left(x_{s}\right)$. Hence there exists a natural map

$$
\tau: \mathcal{Z}_{G}\left(x_{s}\right) *_{P\left(x_{s}\right)} \mathfrak{n}_{2}\left(x_{s}\right) \rightarrow \overline{\mathcal{Z}_{G}\left(x_{s}\right) \cdot x_{n}}, \quad g * n \mapsto g n,
$$

which is a resolution of the singularity of the cone $\overline{\mathcal{Z}_{G}\left(x_{s}\right) \cdot x_{n}}$. The resolution of the $\overline{G(x)}$ is obtained by considering the fibration $F$ over the orbit $G\left(x_{s}\right)$ whose fiber over $x_{s}$ is $\mathcal{Z}_{G}\left(x_{s}\right) *_{P\left(x_{s}\right)} \mathfrak{n}_{2}\left(x_{s}\right)$. The map $\tau$ extends to a map $\tilde{\tau}: F \rightarrow \overline{G(x)}$ as follows. Denote by $\left(x_{s}, y\right)$ the point in the fiber over $x_{s} \in G\left(x_{s}\right)$ in $F$ that is defined by $y \in \mathcal{Z}_{G}\left(x_{s}\right) *_{P\left(x_{s}\right)} \mathfrak{n}_{2}\left(x_{s}\right)$. Then we set $\tilde{\tau}\left(x_{s}, y\right):=\tau_{x_{s}}(y)$.

The resolvable smooth structure $C_{2}^{\infty}(\overline{G(x)})$ is defined by $\tilde{\tau}$ as in Example 2.123. By Lemma $2.16 C_{2}^{\infty}(\overline{G(x)})$ is locally smoothly contractible.

3. In addition, now we assume that $x_{s}=0$, so $\mathcal{Z}_{G}\left(x_{s}\right)=G, P\left(x_{s}\right)=P$ and $\mathfrak{n}_{2}\left(x_{s}\right)=\mathfrak{n}_{2}$. In this case it has been shown in [26] that $\tau^{*}(\omega)$ is a smooth 2-form on $G *_{P} \mathfrak{n}_{2}$. It follows that $C_{2}^{\infty}(\overline{G(x)})$ is weakly symplectic. Panyushev also showed that $\tau^{*}(\omega)$ is symplectic if and only if $x$ is even. (We refer the reader to [6] and [7] for a detailed description of nilpotent orbits.)

Lemma 3.10. Assume that $X$ is a stratified symplectic space with isolated conical singularities and $(\tilde{X}, \tilde{\omega}, \pi: \tilde{X} \rightarrow X)$ a smooth resolution of $X$ such that $\tilde{\omega}$ is a symplectic form on $\tilde{X}$ and $\pi^{*}\left(\omega_{\mid X^{\text {reg }}}\right)=\tilde{\omega}_{\mid \pi^{-1}\left(X^{r e g}\right)}$. If for each singular point $x \in X$ the preimage $\pi^{-1}(x)$ is a coisotropic submanifold in $\tilde{X}$, then the obtained resolvable smooth structure $C^{\infty}(X)$ is Poisson.

Proof. We define a Poisson bracket on $C^{\infty}(X)$ by setting $\{g, f\}_{\omega}(x):=\left\{\pi^{*} g, \pi^{*} f\right\}_{\tilde{\omega}}(\tilde{x})$, for $\tilde{x} \in \pi^{-1}(x)$. We will show that this definition does not depend on the choice of a particular $\tilde{x}$. By definition $\left\{\pi^{*} g, \pi^{*} f\right\}_{\tilde{\omega}}(\tilde{x}):=G_{\tilde{\omega}}\left(d \pi^{*} g, d \pi^{*} f\right)(\tilde{x})$. 
Since $\pi^{*} f$ and $\pi^{*} g$ are constant along the coisotropic submanifold $\pi^{-1}(x)$, we get $G_{\tilde{\omega}}\left(d \pi^{*} g, d \pi^{*} f\right)(\tilde{x})=0$. This proves Lemma 3.10

It has been showed in [1, §2], 26] that the preimage $\tau^{-1}\left(\overline{\mathcal{Z}_{G}\left(x_{s}\right) \cdot x_{n}} \backslash \mathcal{Z}_{G}\left(x_{s}\right)\right.$. $\left.x_{n}\right)$ is a Lagrangian submanifold in $\mathcal{Z}_{G}\left(x_{s}\right) *_{P\left(x_{s}\right)} \mathfrak{n}_{2}\left(x_{s}\right)$ which is the cotangent bundle $T^{*}\left(\mathcal{Z}_{G}\left(x_{s}\right) / P\left(x_{s}\right)\right)$ supplied with the natural symplectic structure. Using Lemma 3.10 we summarize our examination of Example 3.9 in the following

Proposition 3.11. Let $x=x_{n}+x_{s}$ where $x_{n}$ is a minimal nilpotent element in $\mathcal{Z}_{\mathfrak{g}}\left(x_{s}\right)$. Then $C_{1}^{\infty}(\overline{G(x)})$ is a weakly symplectic and Poisson smooth structure. If $x_{s}=0$, then $C_{2}^{\infty}(\overline{G(x)})$ is a weakly symplectic and Poisson smooth structure.

3.3. The existence of Hamiltonian flows. Let $(X, \omega)$ be a stratified symplectic space and $C^{\infty}(X)$ a Poisson smooth structure on $X$. For any $H \in C^{\infty}(X)$ we define a linear operator $X_{H}: C^{\infty}(X) \rightarrow X^{\infty}(X)$ by

$$
X_{H}(f):=\{f, H\}_{\omega} \text { for } f \in C^{\infty}(X) .
$$

By definition, for given $H$, the value $X_{H}(f)(x)$ depends only on the value $d f(x)$. Hence $X_{H}$ is a section of the Zariski tangent bundle of $X$. We call $X_{H}$ the Hamiltonian vector field associated with $H$.

Lemma 3.12. The Hamiltonian vector field $X_{H}$ is a smooth Zariski vector field on $X$. If $x$ is a point in a stratum $S$, then $X_{H}(x) \in T_{x} S$.

Proof. By definition of a Poisson structure, the function $X_{H}(f)$ is smooth for all $f \in C^{\infty}(X)$. Hence $X_{H}$ is a smooth Zariski vector field. This proves the first assertion of Lemma 3.12. To prove the second assertion it suffices to show that, if the restriction of a function $f \in C^{\infty}(X)$ to a neighborhood $U_{S}(x) \subset S$ of a point $x \in S$ is zero, then $X_{H}(f)(x)=0$. The last identity holds, since $X_{H}(f)(x)$ is equal to the Poisson bracket of the restriction of $H$ and $f$ to $S$. This completes the proof.

Theorem 3.13. (cf. 25, §3]) Given a Hamiltonian function $H \in C^{\infty}(X)$ and a point $x \in X$ there exists a unique smooth curve $\gamma:(-\varepsilon, \varepsilon) \rightarrow X$ such that for any $f \in C^{\infty}(X)$ we have

$$
\frac{d}{d t} f(\gamma(t))=\{f, H\}
$$

Proof. Let $\Phi_{t}(x)$ be the flow that is generated by $\left.X_{H}\right|_{S}$ on each stratum $S \subset X$. Since $C^{\infty}(X)$ is Poisson, the validity of (3.3) for $\gamma(t):=\Phi_{t}$ follows from Lemma 3.12. This proves the existence of a flow satisfying (3.3).

Now let us prove the uniqueness of the flow satisfying (3.3), using Sjamaar's and Lerman's argument in [25, §3]. Let $x \in X$ and $\gamma(t), t \in\left(-\varepsilon_{1}, \varepsilon_{1}\right)$ be an integral curve of the equation (3.3) with $\gamma_{0}(0)=x$. We will show that $\Phi_{-t}(\gamma(t))=x$ for all $0 \leq t \leq \min \left(\varepsilon, \varepsilon_{1}\right)$. By Corollary 2.9 smooth functions on $X$ separate points. Therefore it suffices to show that for all $t \leq \min \left(\varepsilon, \varepsilon_{1}\right)$ and for all $f \in C^{\infty}(X)$ we have

$$
f\left(\Phi_{-t}\left(\gamma_{t}(t)\right)\right)=f(x) .
$$

As in [25, §3], using (3.3), we have

$$
\frac{d}{d t} f\left(\Phi_{-t}(\gamma(t))\right)=\{H, f\}_{\omega}(\gamma(t))+\{f, H\}_{\omega}(\gamma(t))=0 .
$$

This implies (3.4) and completes the proof of Theorem 3.13 . 
Remark 3.14. 1. In Example 3.5 we proved that the smooth structure on a singular symplectic reduced space $\left(M^{2 n}, \omega\right) / / G$ defined by Sjamaar and Lerman in [25] is a Poisson smooth structure in sense of our definition. Thus, their result on the existence of a Hamiltonian flow on $\left(M^{2 n}, \omega\right) / / G$ in [25, §3] is a consequence of our Theorem 3.13

2. In [25] Sjamaar and Lerman used a slightly different method for their proof of the existence of a Hamiltonian flow on the singular symplectic reduced space $\left(M^{2 n}, \omega\right) / / G$. They looked at the corresponding Hamiltonian flow on $M$ and showed that this flow descends to a Hamiltonian flow on the reduced space.

3.4. Brylinski-Poisson homology. In this subsection we extend the study of the Brylinski-Poisson homology of symplectic pseudomanifolds with isolated conical singularities in [16, §4] to the case of stratified symplectic spaces $X$ equipped with a Poisson smooth structure.

Assume that $C^{\infty}(X)$ is a Poisson smooth structure. We consider the canonical complex

$$
\rightarrow \Omega^{n+1}(X) \stackrel{\delta}{\rightarrow} \Omega^{n}(X) \rightarrow \ldots
$$

where $\delta$ is a linear operator defined as follows. Let $\alpha \in \Omega(X)$ and $\alpha=\sum_{j} f_{0}^{j} d f_{1}^{j} \wedge$ $d f_{p}^{j}$ be a local representation of $\alpha$ as in Definition 2.10, Then we set (see [12, [3]):

$$
\begin{aligned}
& \delta\left(f_{0} d f_{1} \wedge \cdots \wedge d f_{n}\right):=\sum_{i=1}^{n}(-1)^{i+1}\left\{f_{0}, f_{i}\right\}_{\omega} d f_{1} \wedge \cdots \wedge \widehat{d f_{i}} \wedge \cdots \wedge d f_{n} \\
& +\sum_{1 \leq i<j \leq n}(-1)^{i+j} f_{0} d\left\{f_{i}, f_{j}\right\}_{\omega} \wedge d f_{1} \wedge \cdots \wedge \widehat{d f_{i}} \wedge \cdots \wedge \widehat{d f_{j}} \wedge \cdots \wedge d f_{n}
\end{aligned}
$$

Lemma 3.15. (cf. [16, Lemma 4.3]) $1 . \delta=i\left(G_{\omega}\right) \circ d-d \circ i\left(G_{\omega}\right)$. In particular, $\delta$ is well-defined.

2. $\delta^{2}=0$.

Proof. Recall that $i$ denotes the canonical inclusion $X^{\text {reg }} \rightarrow X$.

1. Let $\alpha \in \Omega(X)$, then $i^{*} \alpha \in \Omega_{u}\left(X^{r e g}\right)$. Using

$$
\delta \circ i^{*}=i^{*} \circ \delta, i^{*} \circ d=d \circ i^{*},
$$

and the validity of the first assertion of Lemma 3.15 for any smooth Poisson manifold $M$ [3, Lemma 1.2.1], we have

$$
i^{*}(\delta \alpha)=\delta\left(i^{*} \alpha\right)=i^{*}\left(i\left(G_{\omega}\right) \circ d \alpha-d \circ i\left(G_{\omega}\right) \alpha\right) .
$$

By Lemma 2.11, $i^{*}$ is injective, hence the above equality implies the first assertion of Lemma 3.15.

2. The second statement of Lemma 3.15 is proved in the same way, using the injectivity of $i^{*}$. This completes the proof of Lemma 3.15

The following theorem 3.16 generalizes [16, Corollary 4.2] .

Theorem 3.16. Suppose $(X, \omega)$ is a stratified symplectic space equipped with a Poisson smooth structure $C^{\infty}(X)$ which is also weakly symplectic. If all regular strata of $X$ has the same dimension $2 n$, the Brylinski-Poisson homology of the complex $(\Omega(X), \delta)$ is isomorphic to the de Rham cohomology of $X$ with reverse grading: $H_{k}(\Omega(X), \delta)=H^{2 n-k}(\Omega(X), d)$. If, moreover, the smooth structure $C^{\infty}(X)$ is locally smoothly contractible, $H_{k}(\Omega(X), \delta)$ is equal to the singular cohomology $H^{2 n-k}(X, \mathbb{R})$. 
Proof. Using the injectivity of $i^{*}$, we derive from (3.5) the following formulas for all $k$ :

$$
H_{k}(\Omega(X), \delta)=H_{k}\left(i^{*}(\Omega(X), \delta) \text { and } H^{k}(\Omega(X), d)=H^{k}\left(i^{*}(\Omega(X), d) .\right.\right.
$$

Since $C^{\infty}(X)$ is weakly symplectic, there exists a symplectic form $\tilde{\omega} \in \Omega^{2}(X)$ such that $\left.i^{*}(\tilde{\omega})\right|_{S_{i}}=\omega_{i}$ for any stratum $S_{i}$. Set vol $:=\tilde{\omega}^{n} / n$ !. Let $\tilde{G}_{\omega}^{k}$ be the pairing: $\Lambda^{k}\left(T^{*} X\right) \times \Lambda^{k}\left(T^{*} X\right) \rightarrow C^{\infty}(X)$ associated with $\tilde{G}_{\omega}$ whose existence is shown in Remark 3.4 2. We define a symplectic star operator $*_{\omega}: \Omega^{k}(X) \rightarrow$ $\Omega^{2 n-k}(X)$ as follows (cf. [3, §2.1]).

$$
*_{\omega}: \Omega^{k}(X) \rightarrow \Omega^{2 n-k}(X), \beta \wedge *_{\omega} \alpha:=\tilde{G}_{\omega}^{k}(\beta, \alpha) \wedge \frac{\tilde{\omega}^{n}}{n !},
$$

for all $\alpha, \beta \in \Omega^{k}(X)$. In particular, on singular strata, the image of $*_{\omega}$ is zero.

For the sake of simplicity we also denote by $\omega$ the restriction of $\omega$ to $X^{r e g}$. The following Proposition is proved by repeating the proof of Proposition 4.2 in [16] word-by-word, so we omit its proof.

Proposition 3.17. We have $*_{\omega}\left(i^{*}\left(\Omega^{k}(X)\right)\right)=i^{*}\left(\Omega^{2 n-k}(X)\right)$.

The first assertion of Theorem 3.16 follows immediaately from (3.6) and Proposition 3.17. The second assertion of Theorem 3.16 follows from 23. This completes the proof of Theorem 3.16 .

3.5. A Leftschetz decomposition. The notion of a Leftschetz decomposition on a symplectic manifold $\left(M^{2 n}, \omega\right)$ has been introduced by Yan in [29], where he gives an alternative proof the Mathieu theorem on harmonic cohomology classes of $\left(M^{2 n}, \omega\right)$ using the Leftschetz decomposition. Roughly speaking, a Leftschetz decomposition on a symplectic manifold $\left(M^{2 n}, \omega\right)$ is an $\mathfrak{s l}_{2}$-module -structure of $\Omega\left(M^{2 n}\right)$. The Lie algebra $\mathfrak{s} l_{2}$ acting on $\Omega\left(M^{2 n}\right)$ is generated by linear operators $L, L^{*}, A$ defined as follows. $L$ is the wedge multiplication by $\omega, L^{*}:=i\left(G_{\omega}\right)$, and $A=\left[L^{*}, L\right]$

Now assume that $(X, \omega)$ is a stratified symplectic space provided with a Poisson smooth structure $C^{\infty}(X)$, which is also weakly symplectic. Then $\Omega(X)$ is stable under $L, L^{*}$. Hence we obtain immediately

Lemma 3.18. The space $\Omega(X)$ is an $\mathfrak{s l}_{2}$-module, where $\mathfrak{s l}_{2}$ is the Lie algebra generated by $\left(L, L^{*}, A=\left[L, L^{*}\right]\right)$.

The next Lemma concerns the Leftschetz decomposition of $\Omega_{u}\left(X^{r e g}\right)$. Recall that $\alpha \in \Omega_{u}\left(X^{r e g}\right)$ is called primitive, if $L^{*} \alpha=0$. We define the dimension function $\mathrm{d}: X \rightarrow \mathbb{Z}$ by setting $\mathrm{d}(x):=\operatorname{dim} S_{x}$, where $S_{x}$ is the connected component of the stratum containing $x$.

Lemma 3.19. 1. For any $\gamma \in \Omega_{u}^{k}\left(X^{\text {reg }}\right)$ and any $r \in \mathbb{Z}$ we have

$$
\left[L^{r}, L^{*}\right] \gamma=\left(r\left(k-\frac{\mathrm{d}}{2}\right)+r(r-1)\right) L^{r-1} .
$$

2. There exists a function $c: \mathbb{Z} \times \mathbb{Z} \rightarrow \mathbb{R}$ such that for any primitive form $\gamma \in \Omega\left(X^{r e g}\right)$ we have $\gamma=c(\mathrm{~d}, k) \cdot\left(L^{*}\right)^{k} \circ\left(L^{k} \gamma\right)$.

Proof. The first assertion of Lemma 3.19 for $r=1$ is well-known, see 29, Corollary 1.6]. For $r \geq 2$ we use the following formula

$$
\left[L^{r}, L^{*}\right]=L\left[L^{r-1}, L^{*}\right]+\left[L, L^{*}\right] L^{r-1},
$$


which leads to the first assertion of Lemma 3.19 by induction.

2. The second assertion of Lemma 3.19 is proved by applying the first assertion recursively.

For any $k \geq 0$ set

$$
\mathcal{P}_{k}(X):=\left\{\alpha \in \Omega^{k}(X) \mid \alpha \text { is primitive }\right\} .
$$

Proposition 3.20. Assume that $C^{\infty}(X)$ is both Poisson and weakly symplectic. If all regular strata of $X$ have the same dimension $2 n$, then we have the following Leftschetz decomposition for $k \geq 0$

$$
\Omega^{k}(X)=\mathcal{P}_{k}(X) \oplus L\left(\mathcal{P}_{k-2}(X)\right) \oplus \cdots
$$

Proof. Using the Leftschetz decomposition on symplectic manifold, for $\alpha \in \Omega^{k}(X)$ we decompose $i^{*}(\alpha) \in i^{*}\left(\Omega^{k}(X)\right)$ as

$$
i^{*}(\alpha)=\alpha_{p}^{k}+L\left(\alpha_{p}^{k-2}\right)+\cdots+L^{[(n-k) / 2]} \alpha_{p}^{n-k-2[(n-k) / 2]},
$$

where $\alpha_{p}^{j}$ are primitive forms in $\Omega_{u}^{j}\left(X^{r e g}\right)$. To prove Proposition 3.20 using the injectivity of $i^{*}$, it suffices to show that $\alpha_{p}^{j}$ are elements in $i^{*}(\Omega(X))$. Now let us consider the decomposition of $i^{*}(\alpha) \in \Omega_{u}^{k}\left(X^{r e g}\right)$. We will show that all terms $\alpha_{p}^{j}$ can be obtained from a linear combination of $L^{p}\left(i^{*}(\alpha)\right),\left(L^{*}\right)^{p}\left(i^{*}(\alpha)\right), p \geq 0$, inductively on the degree $j$.

First we assume that $k$ is even, i.e. $k=2 q$, hence $\alpha_{p}^{0} \in C_{u}^{\infty}\left(X^{r e g}\right)$. Applying to the both sides of (3.7) the operator $L^{n-q}$ we get

$$
L^{n-q}\left(i^{*}(\alpha)\right)=\omega^{n} \cdot \alpha_{p}^{0} \in i^{*}\left(\Omega^{2 n}(X)\right) .
$$

By Proposition 3.17 (3.8) implies that $\alpha_{p}^{0} \in i^{*}\left(C^{\infty}(X)\right)$, what is required to prove in the first induction step.

Now let us assume that $k=2 q+1$. As in (3.8), we have

$$
L^{n-q-1}\left(i^{*}(\alpha)\right)=\omega^{n-1} \cdot \alpha_{p}^{1} \in i^{*}\left(\Omega^{2 n-1}(X)\right) .
$$

Taking into account $L^{*} \alpha_{p}^{1}=0$, using Lemma 3.19 and (3.9), the term $\alpha_{p}^{1}$ can be obtained from $L^{n-q-1}\left(i^{*}(\alpha)\right)$ by applying the operator $c_{n, 1} \cdot\left(L^{*}\right)^{n-1}$. Hence $\alpha_{p}^{1} \in i^{*}\left(\Omega\left(X^{2 n}\right)\right)$, which completes the next induction step.

Repeating this procedure, we get all terms $\alpha_{p}^{j}$, which belong to $i^{*}(\Omega(X))$. This completes the proof of Proposition 3.20

Since $[L, d]=0$ holds on $\Omega_{u}\left(X^{r e g}\right)$ and $i^{*}(\Omega(X))$ is stable under the action of $d$ and $L$, the equality $[L, d]=0$ also holds on $\Omega(X)$. In particular, the wedge product with $\left[\omega^{k}\right]$ maps $H^{l-k}(\Omega(X), d)$ to $H^{l+k}(\Omega(X), d)$ for any $l \in \mathbb{Z}$. A stratified symplectic space $\left(X^{2 n}, \omega\right)$ of dimension $2 n$ equipped with a Poisson weakly symplectic smooth structure $C^{\infty}\left(X^{2 n}\right)$ is said to satisfy the hard Lefschetz condition, if the cup product

$$
\left[\omega^{k}\right]: H^{n-k}\left(\Omega\left(X^{2 n}\right), d\right) \rightarrow H^{n+k}\left(\Omega\left(X^{2 n}\right), d\right)
$$

is surjective for any $k \leq n=\frac{1}{2} \operatorname{dim} X^{2 n}$. A differential form $\alpha \in \Omega\left(X^{2 n}\right)$ is called harmonic, if $d \alpha=0=\delta \alpha$. Let us abbreviate $H^{*}\left(\Omega\left(X^{2 n}\right), d\right)$ by $H_{d R}^{*}\left(X^{2 n}\right)$.

Theorem 3.21. Let $\left(X^{2 n}, \omega\right)$ be a stratified symplectic space and $C^{\infty}\left(X^{2 n}\right)$ Poisson smooth structure which is also weakly synmplectic. Assume that all regular strata of $X$ have the same dimension $2 n$. Then the following two assertions are equivalent: 
(1) Any cohomology class in $H_{d R}^{*}\left(X^{2 n}\right)$ contains a harmonic cocycle.

(2) $\left(X^{2 n}, \omega\right)$ satisfies the hard Lefschetz condition.

Proof. The proof of Theorem 3.21 for smooth symplectic manifolds by Yan in [29, Theorem 0.1] can be repeated word-by-word. For the convenience of the reader we outline a proof here. Denote by $H_{h r}^{k}\left(X^{2 n}\right)$ the space of all harmonic $k$-forms on $\left(X^{2 n}, \omega\right)$, and let $H_{h r}^{*}=\oplus_{i=0}^{2 n} H_{h r}^{i}\left(X^{2 n}\right)$.

Now let us prove that the assertion (1) of Theorem 3.16 implies the assertion (2) of Theorem 3.16. We consider the following diagram

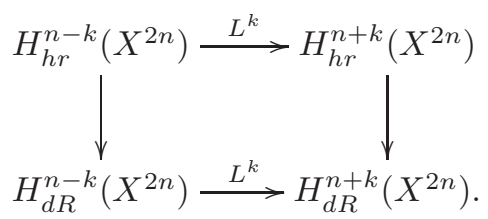

Let us recall that $i: X^{r e g} \rightarrow X^{2 n}$ is the canonical inclusion. Since $[L, \delta]=-d[29$, Lemma 1.2], which can be easily proved for $\left(X^{2 n}, \omega\right)$ satisfying the condition of Theorem 3.21. Proposition 3.20 implies that $L^{k}: H_{h r}^{n-k}\left(X^{2 n}\right) \rightarrow H_{h r}^{n+k}\left(X^{2 n}\right)$ is an isomorphism. Since the vertical arrows in the diagram are surjective, we conclude that the second horizontal arrow in the diagram is also surjective. This proves (1) $\Longrightarrow(2)$.

Now let us prove that $(2) \Longrightarrow(1)$. Note that the condition (2) implies that 29 , $\S 3]$

$$
H_{d R}^{n-k}\left(X^{2 n}\right)=\operatorname{Im} L+P_{n-k},
$$

where $P_{n-k}:=\left\{\alpha \in H_{d R}^{n-k}\left(X^{2 n}\right) \mid L^{k+1} \alpha=0 \in H_{d R}^{n+k+2}\left(X^{2 n}\right)\right\}$.

Using induction argument, it suffices to prove that in each primitive cohomology class $v \in P_{n-k}$ there is a harmonic cocycle. Let $v=[z], z \in \Omega^{n-k}\left(X^{2 n}\right)$. Since $v$ is primitive we have $\left[z \wedge \omega^{k+1}\right]=0 \in H_{d R}^{m+k+2}\left(X^{2 n}\right)$. Hence, $z \wedge \omega^{k+1}=d \gamma$ for some $\gamma \in \Omega^{n+k+1}\left(X^{2 n}\right)$. By Proposition 3.20 the operator $L^{k+1}: \Omega^{n-k-1}\left(X^{2 n}\right) \rightarrow$ $\Omega^{n+k+1}\left(X^{2 n}\right)$ is onto, consequently there exists $\theta \in \Omega^{m-k-1}\left(X^{2 n}\right)$ such that $\gamma=$ $\theta \wedge \omega^{k+1}$. It follows that $(z-d \theta) \wedge \omega^{k+1}=0$. Therefore $w=z-d \theta$ is primitive and closed. Since $\left[L^{*}, d\right]=\delta[29$, Corollary 1.3], we obtain $\delta w=0$. This completes the proof of Theorem 3.16 .

Remark 3.22. 1. If $C^{\infty}\left(X^{2 n}\right)$ is locally smoothly contractible, by 23, Theorem 5.2] the de Rham cohomology $H^{*}\left(\Omega\left(X^{2 n}\right), d\right)$ coincides with the singular cohomology $\left.H^{*}(M, \mathbb{R})\right)$, since $X^{2 n}$ admits smooth partitions of unity, see Proposition 2.8 .

2. In [4, Proposition 5.4] Cavalcanti proved that the hard Lefschetz property on a compact symplectic manifold implies $\operatorname{Im} \delta \cap \operatorname{ker} d=\operatorname{Im} d \cap \operatorname{Im} \delta$, see also [22]. His theorem can be proved word-by-word for stratified symplectic spaces satisfying the conditions of Proposition 3.20 since the main ingredient of the proof is Proposition 3.20 .

3. It is interesting to know whether we can extend the symplectic cohomology theory developed in 28, 17 to stratified symplectic spaces satisfying the conditions of Proposition 3.20, since the main ingredient of this theory is the existence of a Leftschetz decomposition. 


\section{Conclusions}

In this paper we have worked out a natural refinement of the concept of a smooth structure on a stratified space, which is well suited for the study of stratified symplectic spaces. In this refined concept there are two natural classes of smooth structures on stratified symplectic spaces: weakly symplectic smooth structures and Poisson smooth structures. We show that Poisson smooth structures on stratified symplectic spaces $X$, especially those are also weakly symplectic, enjoy many properties of symplectic manifolds, if the regular strata of $X$ are of the same dimension. We suggest to study stratified symplectic spaces, whose regular strata are of varying dimension, for future works.

Funding This work was supported by RVO: 67985840 and by Grant of ASCR Nr100190701 (H.V. L. and J. V.), by MSM 0021620839 and GACR 20108/0397 (P.S.). A part of this paper was written while H.V.L. was visiting the ASSMS, GCU, Lahore-Pakistan. She thanks ASSMS for their hospitality and financial support.

Acknowledgement H.V.L. thanks Dmitry Panyushev and Nguyen Tien Zung for discussions, which have helped to shape the final form of this paper.

\section{REFERENCES}

[1] A. Beauville, Symplectic singularities, Invent. Math. 139 (2000), 541-549.

[2] L. Bates and E. Lerman, Proper group actions and stratified symplectic spaces, Pacific Journal of Math., 181(1997), 201-229.

[3] J.C. Brylinksi, A differential complex for Poisson manifolds, JDG 28 (1988), 93-114.

[4] G. R. Cavalcanti, New aspects of the $d d^{c}$-lemma, PhD Thesis, Oxford 2005.

[5] W. Chen and Y. Ruan, Orbifold Gromov-Witten theory, In the book: Orbifolds in mathematics and physics, (Madison, WI, 2001), 25-85, Contemp. Math., 310, Amer. Math. Soc., Providence, RI, 2002.

[6] D. H. Collingwood and W. M. McGovern, Nilpotent Orbits in Semisimple Lie Algebras, Van Nostrand Reinhold, 1993, New York.

[7] B. Fu, A survey on symplectic singularities and symplectic resolutions, Ann. Math. Blaise Pascal 13 (2006), no. 2, 209-236.

[8] B. Fu, Symplectic resolution for nilpotent orbits, Inv. Math. 151 (2003), 167-186.

[9] M. Goresky and R. MacPherson, Intersection homology theory, Topology 19 (1980), $135-162$.

[10] M. Goresky and R. MacPherson, Stratified Morse theory, Springer Verlag, New York 1988.

[11] J. A. N. GonzÁlez and J.B.S. de Salas, $C^{\infty}$-differentiable spaces, Lecture Notes in Mathematics, 1824, Springer-Verlag, Berlin, 2003.

[12] J.L. Kozsul, Crochet de Schouten-Nijenhuis et cohomologie, in "Elie Cartan et les Math. d'Aujour d'Hui", Asterisque hors-serie, 1985, 251-171.

[13] J. Huebschmann, Kähler spaces, nilpotent orbits, and singular reduction, Memoirs of the AMS, 172 (814) (2004), Amer. Math. Soc., Providence R.I., math.DG/0104213

[14] J. Huebschmann, G. Rudolph and M. Schmidt, A gauge model for quantum mechanics on a stratified space, Commun. Math. Physics, 286 (2009), 459-494, hep-th/0702017

[15] Y. KARSHON, An algebraic proof for the symplectic structure of moduli space, Proceedings of AMS, 116(1992), 591-605.

[16] H. V. Lê, P. Somberg and J. Vanzura, Smooth structures on conical pseudomanifolds, Acta Mathematica Vietnamica, 38 (2013) n 1, 33-54, arXiv:1006.5707

[17] H.V. Lê, AND J. VANŽUra, Cohomology theories on locally conformally symplectic manifolds, arXiv:1111.3841.

[18] H. Matsumura, Commutative algebra, Benjamin/Cummings, London 1980.

[19] J. N. Mather, Note on topological stability, Mimeographed Lecture Notes, Harvard, 1970. 
[20] O. Mathieu, Harmonic cohomology classes of symplectic manifolds, Comment. Math. Helv. 70 (1995), 1-9.

[21] I. MoerdiJk and G. E. Reyes, Models for smooth infinitesimal analysis, Springer-Verlag, New York 1991.

[22] S. A. Merkulov, Formality of canonical symplectic complexes and Frobenius manifolds, Internat. Math. Res. Notices (1998), no. 14, 727-733.

[23] M. Mostow, The differentiable structure of Milnor classifying spaces, simplicial complexes, and geometric realizations, J. D. G. 14 (1979), 255-293.

[24] V. E. Nazaikinskit, A. Yu. Savin, B. Yu. Sternin, B. V. Shultse, On the existence of elliptic problems on manifolds with edges. (Russian) Dokl. Akad. Nauk 395 (2004), no. 4, $455-458$.

[25] R. Sjamaar, and E. Lerman, Stratified Spaces and Reduction, Ann. of Math., 134 (1991), p.375-422.

[26] D. Panyushev, Rationality of singularities and the Gorenstein properties of nilpotent orbits, Functional Anal. Appl. 25 (1991), 225-226.

[27] M. J. Pflaum, Analytic and geometric study of stratified spaces. Lecture Notes in Mathematics, 1768. Springer-Verlag, Berlin, 2001.

[28] L.S Tseng and S.T Yau, Cohomology and Hodge Theory on Symplectic Manifolds, I, II arXiv:0909.5418, arXiv:1011.1250

[29] D. YAn, Hodge Structure on Symplectic Manifolds, Advances in Math. 120 (1996), 143154.

Hông Vân Lê, Institute of Mathematics of ASCR, Zitna 25, 11567 Praha 1, Czech Republic

Petr Somberg, Mathematical Institute, Charles University, Sokolovska 83, 18000 Praha 8, Czech Republic.

Jiři Vanžura, Institute of Mathematics of ASCR, Zizkova 22, 61662 Brno, Czech Republic 